\title{
Improving the percentage of electronic discharge summaries completed within 24 hours of discharge
}

Michael Haycock, Laura Stuttaford, Oliver Ruscombe-King, Zoe Barker, Kathryn Callaghan, Timothy Davis

Great Western Hospital, UK

\begin{abstract}
EDSs are an important part of patient care and medical communication. The GWH has a financially motivated target stating that $95 \%$ of EDS are to be completed within 24 hours of patient discharge. On review of a six-week pre-intervention period, the medical ward mean weekly EDS completion rate within 24 hours was $74.3 \%$.

EDSs form a significant part of junior doctor workload. We found that on a medical ward the mean completion time for one EDS was 18.25 minutes. In January 2014, 387 EDSs were written between four medical wards. This equates to 29.25 hours per week of junior doctor time spent completing EDSs on the four main medical wards.
\end{abstract}

Our aim was to improve the percentage of EDSs completed within 24 hours of discharge from medical wards in the GWH.

We proposed and implemented two interventions:

1) Five day EDS summary

2) Protected EDS hour.

The five day EDS summary was implemented on wards 1 and 2. The protected EDS hour on ward 3.

Ward 1: mean pre-intervention EDS completion rate: $81.1 \%$ (six months pre-intervention). This increased by $7.9 \%$ to $89 \%$ (four week mean EDS completion rate post-intervention)

Ward 2: mean pre-intervention EDS completion rate: $75.2 \%$. This increased by $11.6 \%$ to $86.8 \%$

Ward 3: mean pre-intervention EDS completion rate: $71 \%$. This increased by $4.5 \%$ to $75.5 \%$

Control ward: mean pre-intervention EDS completion rate: $85.1 \%$. This increased by $5.1 \%$ to $90.2 \%$

Our results show the five day EDS summary led to a mean $9.75 \%$ improvement and the protected EDS hour a mean $4.5 \%$ improvement in EDS completion rates. A $5.1 \%$ increase was seen on the control ward suggesting confounding factors in this data which are most likely the trust EDS working group, junior doctor experience and EDS project publicity.

\section{Problem}

Communication between the hospital and primary care is essential once a patient has been discharged in order to inform the community team of the reason for admission and any postdischarge plans. Discharge summaries are one of the main tools utilised to achieve this. Healthcare professionals in the community feel that timely discharge summaries with sufficient information are essential. However, discharge summaries are not always received early enough and are sometimes lacking in important information.[1]

The use of electronic discharge summaries (EDSs) as opposed to the antiquated hand written versions can reduce or eliminate the problems caused by illegibility, incompleteness and inaccuracies. It is also much easier to trace whether a discharge summary has been completed and how promptly it was received in primary care. CQUIN targets state that a proportion of a Trust's discharge summaries should be received within 24 hours of discharge. This is described in the toolkit produced by the Health and Social Care Information Centre.[2]

The Great Western Hospital (GWH), Swindon, UK, has a financially motivated target stating that $95 \%$ of EDSs are to be completed within 24 hours of patient discharge. On review of a six-week preintervention period $(22 / 7 / 13-1 / 9 / 13)$, the medical ward mean weekly EDS completion rate within 24 hours was $74.3 \%$. This data was collected by the hospital's EDS working group and was taken from all medical wards (excluding outliers). 


\section{Background}

One ward within the hospital was regularly attaining a 100\% EDS submission rate within 24 hours and the staff (including the ward clerk and junior doctors) were asked to comment on how they thought this was being achieved. The main reasons given were that some EDSs were started before discharge, junior doctors were released from ward round by the consultant to complete an EDS, and there was good communication between members within the multidisciplinary team.

We distributed a junior doctor questionnaire to investigate the issues that were causing this poor performance. The overwhelming response was that time pressures and poor communication were the main causes of late EDSs.

We undertook an audit into EDS workload. We found that on a medical ward the mean completion time for one EDS was 18.25 minutes (n: 20). In January 2014, 387 EDSs were written on the four medical wards. This equates to 29.25 hours per week of junior doctor time spent completing EDSs or roughly four hours per medical team.

We further investigated why EDSs were late using data from a one week period in unscheduled care. Within this week, eight EDS were late of which $38 \%$ were actually written on time but not submitted to the Medway system. Additionally, $37 \%$ of these EDSs belonged to deceased patients, and the remaining $25 \%$ had no reason identified.

\section{Baseline measurement}

The Trust EDS working group collected weekly data on the percentage of EDSs being completed within 24 hours of patient discharge.

\section{Design}

Our aim was to improve the percentage of EDSs completed within 24 hours of discharge from the medical wards at the GWH. We proposed two interventions. Firstly, to promote a five day EDS summary. We hypothesized that a partially complete EDS would take less time to complete than producing the whole EDS. We planned that after five days of patient stay, junior doctors would be prompted to start the EDS. It was anticipated that a partially complete EDS would also help timely completion if the patient moves wards or medical teams, as the new junior doctor would not have to complete a full EDS for an unfamiliar patient which would typically involve wading through a large stack of inpatient notes. Our hypothesis would be supported by an improvement in the weekly percentage of EDSs completed on time.

The second intervention was the promotion of a protected EDS hour. We hypothesized that junior doctors would be more productive at writing EDSs if they are not interrupted. We suggested that one junior doctor, per medical team, per day, should be allowed to have one hour free of routine ward jobs to complete EDSs.
Another member of the medical team would cover routine wards jobs during this time. The nursing staff would be encouraged to contact another doctor, except in emergencies, to allow the EDS hour to remain free of distractions. Again, we looked for improvement in the weekly percentage of EDSs completed on time.

\section{Strategy}

PDSA cycle 1: On ward 4 (a respiratory ward) we introduced the use of a five day summary and on ward 1 (a cardiology/endocrinology ward) we introduced a protected EDS hour. Junior doctors on both of the wards were notified of the changes. Unfortunately, the junior doctors on ward 4 were too busy to engage with the project. On ward 1, some consultants did not think the project would be beneficial as they felt their junior doctors have plenty of time to write discharge summaries. Also, each junior doctor on ward 1 is allocated their own patients which made crosscovering for the EDS hour very difficult. From doing the first cycle we identified the importance of tailoring interventions to each ward and ensuring consultants are involved.

PDSA cycle 2: For the second cycle we tailored our intervention to specific wards, allocated junior doctors from the EDS group to each ward, ensured consultants were supportive of the interventions, and increased the promotion of our project. During this cycle we introduced the use of the five day summary on wards 1 and 2 (an elderly care ward) and introduced the one hour protected EDS time on ward 3 (a gastroenterology ward).

\section{Post-measurement}

On ward 1, the mean pre-intervention EDS completion rate was $81.1 \%$ (six months pre-intervention) within 24 hours of discharge. This increased by $7.9 \%$ to $89 \%$ (four week mean EDS completion rate post-intervention) (see run chart).

On ward 2, the mean pre-intervention EDS completion rate was $75.2 \%$ (six months pre-intervention). This increased by $11.6 \%$ to 86.8\% (four week mean EDS completion rate post-intervention) (see run chart).

On ward 3, the mean pre-intervention EDS completion rate was $71 \%$ (six months pre-intervention). This increased by $4.5 \%$ to $75.5 \%$ (four week mean EDS completion rate post-intervention) (see run chart).

On the control ward, the mean pre-intervention EDS completion rate was $85.1 \%$ (six months pre-intervention). This increased by $5.1 \%$ to $90.2 \%$ (four week mean EDS completion rate postintervention) (see run chart).

Our results show the five day EDS summary led to a mean $9.75 \%$ improvement and the protected EDS hour a mean $4.5 \%$ improvement in EDS completion. There was also an improvement in the significant variation in weekly EDS completion rates postintervention across all wards including the control ward (see run chart). A $5.1 \%$ increase in EDS completion rates was seen on the 


\section{BMJ Quality Improvement Reports}

control ward suggesting significant confounding factors in this data.

Mark Juniper, Susan Chalstrey, Jessica Daniel, and Carolyn Bell.

See supplementary file: ds4266.docx - "Run charts showing the percentage of EDSs completed on time on a week to week basis from the trial wards (second PDSA)"

\section{Lessons and limitations}

This was a challenging project. Junior doctors found it difficult to engage with our project due to their workload and we noticed a ward culture that is generally resistant to change. Consultant backing was essential to the success of our proposed interventions. We learnt that consultants are a key resource when trying to improve and change the culture among staff on hospital wards.

There are a large number of confounding factors in our data. Most notably the work done by the Trust's EDS working group to improve the percentage completed within the required time frame. Other confounding factors include junior doctor experience and EDS project publicity. More junior doctors became aware of the importance of completing an EDS within 24 hours of discharge thanks to the publicity of this project, and we also highlighted the importance of electronically sending the EDS on completion as it does not count in the figures as "on time" unless it has been sent to the computer system.

\section{Conclusion}

EDSs are an important part of patient care and medical communication. It is therefore important that they are completed promptly. Our results show the five day EDS summary led to a mean $9.75 \%$ improvement in EDS completion rates, and the protected EDS hour a mean $4.5 \%$ improvement in EDS completion rates within 24 hours of patient discharge.

However a $5.1 \%$ increase was seen on the control ward which likely reflects the confounding factors previously mentioned. This is a complex, multi-factorial area where there is still a need for more improvement to be made.

\section{References}

1. Hesselink $G$ et al. Are patients discharged with care? A qualitative study of perceptions and experiences of patients, family members and carers. BMJ Quality and Safety 2012; 21:i39-i49. Available from: http://qualitysafety.bmj.com/content/21/Suppl_1/i39.full

2. Department of Health, The Discharge Summary Implementation Toolkit (version 2). November 2011. Available from: http://systems.hscic.gov.uk/clinrecords

\section{Declaration of interests}

Nothing to declare.

\section{Acknowledgements}

